\title{
TECHNOLOGICAL LEVEL OF FLEXIBLE MANUFACTURING SYSTEM CONTROL
}

\author{
Ján JADLOVSKÝ, Stanislav LACIŇÁK, Matej ČOPÍK, Ján ILKOVIČ \\ Department of Cybernetics and Artificial Intelligence, Faculty of Electrical Engineering and Informatics, \\ Technical University of Košice, Letná 9, 04200 Košice, Slovak Republic, tel.: +421 556024218 , \\ e-mail: jan.jadlovsky@tuke.sk, stanislav.lacinak@tuke.sk, matej.copik@tuke.sk,jan.ilkovic@tuke.sk
}

\begin{abstract}
This article describes technological level of flexible manufacturing system (FMS) control. It focuses on the control in terms of sensors, actuators and technological networks. It also focuses on the control of manipulators that are part of FMS and are controllled via the network DeviceNet. Flexible manufacturing system (FMS) represents fully automated technological manufacturing process that is employed in teaching process. The principal aim is to improve the control system of a given model as well as higher levels of distributed control system (DCS) using the communication links between individual DCS levels.
\end{abstract}

Keywords: Flexible Manufacturing System (FMS), DeviceNet, Distributed Control System (DCS), Technological Level of Control, Programmable Logic Controller (PLC), Sensors, Actuators, manipulators

\section{INTRODUCTION}

A new school research and teaching model shown in Fig. 1 is currently being integrated in teaching process at the Department of Cybernetics and Artificial Intelligence at the Technical University of Košice. This model was financed from the Centre of Excellence project.

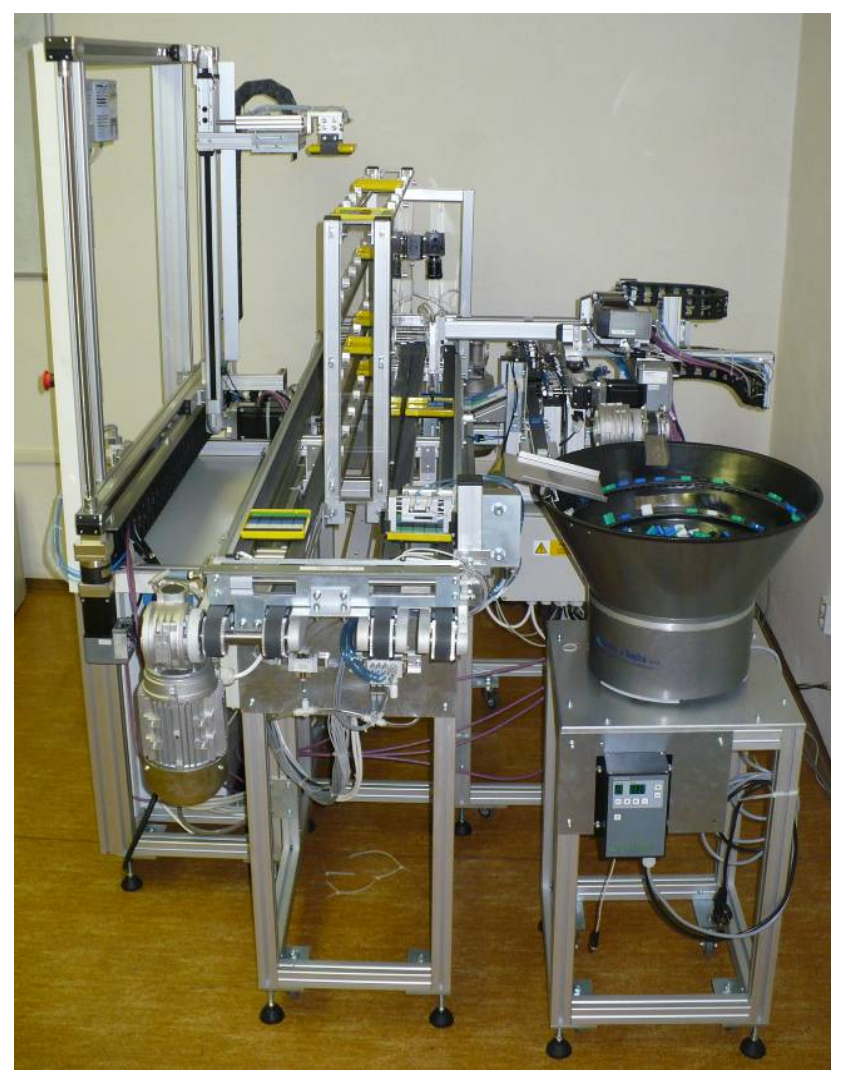

Fig. 1 Flexible manufacturing system in school environment

The FMS model has been designed and assembled in a way that conforms definitions of Distributed control system theory and is located within the department. The model has already provided space for realisation of several theses and is still waiting for many other theses, bachelor's pieces of work or dissertations to be realised with it.

\section{DISTRIBUTED CONTROL SYSTEM}

Distributed control system (DCS) is a control system, usually production system, process or any dynamic system in which the system elements are not located centrally but they are distributed, divided into smaller parts, subsystems that are controlled by one or more control devices/controllers. Current industrial information and control systems (IaRS) utilize mainly hierarchic (pyramidal) architectures containing physical and logical distribution elements, integration as a whole, open and scalable. Intelligent features have been applied on a large scale recently whereby direct hierarchic relations are turned into network relations. Emergent trends have also started to appear to a great degree, i.e. merging of originally independent systems, which can result in their new features generation as a whole. Individual world-wide automation leaders develop their own models that are adapted to their scope and production. The IaRS pyramid shown in figure 2 represents the usual architecture of such systems.

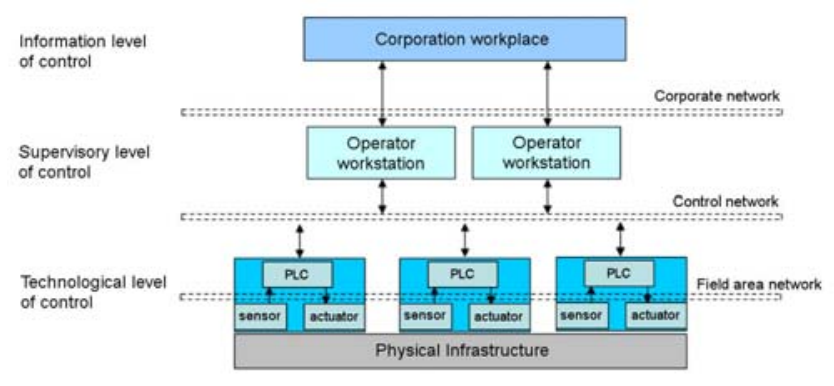

Fig. 2 Control systems architecture

Technological level of control/management represents basic interface with production. It includes production lines and machinery in which sensors and actuators are integrated and these communicate with process control computers, mainly PLCs (Programmable Logic Controllers) through technological networks. Level of 
supervisory management is a higher level of management which is also alternatively called SCADA/HMI level (Supervisory Control and Data Acquisition / Human Machine Interface). It has been established for primary acquisition and integration of process data, monitoring, visualization, evaluation and direct interference in manufacturing processes. Information level of management represents the top management in relation to the previous levels. Here belong database resources for higher levels of management, management information system and means of Internet visualization. Information level of management is a planning and management level. At this level, data is archived and processed, and longterm strategic decisions for production are accepted.

DCS is widely used to control manufacturing processes in such fields as petrochemical, pharmaceutical, power engineering, food processing and metallurgical industry. Therefore it represents the most suitable way how to control the school model of flexible production line.

\subsection{Device Net}

DeviceNet is an industrial network system that allows simple connection to and remote control of a wide range of devices. It has been designed by the company Rockwell Automation. Everything from programmable logic controllers (PLC), remote inputs and outputs to optical fibre sensors, power units and frequency inverters can be fully integrated in the DeviceNet system. DeviceNet simplifies wiring by utilizing dual coiled twin-lead for power supply and data transmission.

DeviceNet is based on open standards and specifications defined by the association of suppliers Open DeviceNet Vendors Association (ODVA). It is a syndicate whose main mission is to support the DeviceNet product on a world-wide scale. All ODVA certified products are fully compatible with the network DeviceNet.

DeviceNet specification is based on the Controller Area Network (CAN) protocol standard. CAN protocol supports two formats of message frames: standard CAN (version 2.0A, 11-bit identifier) and extended CAN (version 2.0B, 29-bit identifier).

Frame format of the CAN protocol for the network DeviceNet is shown in Fig. 3. Total number of additional bits is 47 and they cover the fields: start of frame (SOF), arbitration (11-bit identifier), control, data, CRC, acknowledgment (ACK), end of frame and pause between INT (interframe) transmission. Data block size is within the range 0 up to 8 bytes. CAN protocol utilizes arbitral field to secure source and target address and to resolve message priorities.

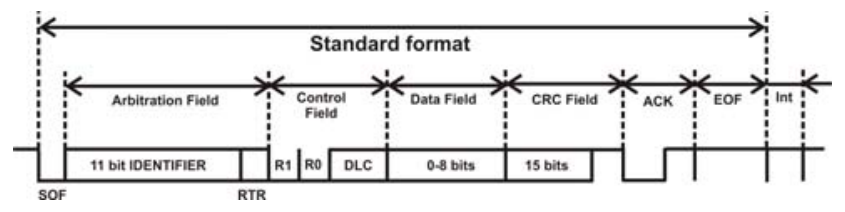

Fig. 3 CAN protocol frame format for DeviceNet network

Technical parameters:

- supports both master/slave and peer-to-peet communication,
- $\quad$ network devices can be controlled and configured at the same time,

- $\quad$ power cord for DeviceNet network can supply voltage and can be used as a communication cable as well,

- $\quad$ topology of DeviceNet network can be of various type (circle, bus, star, tree, mixed),

- bit rate depends on the used cable from $125 \mathrm{kbit} / \mathrm{s}$ up to $500 \mathrm{kbit} / \mathrm{s}$.

\section{DESCRIPTION OF FLEXIBLE MANUFACTURING SYSTEM CONTROL AT TECHNOLOGICAL LEVEL}

Flexible manufacturing system (FMS) project shown in Fig. 4 includes as a whole all parts of distributed control system. Since the overall issue of system control is complicated to a considerable degree, it is divided into several parts. This article deals with connection and control of manipulators and other devices of constructed flexible manufacturing system (FMS).

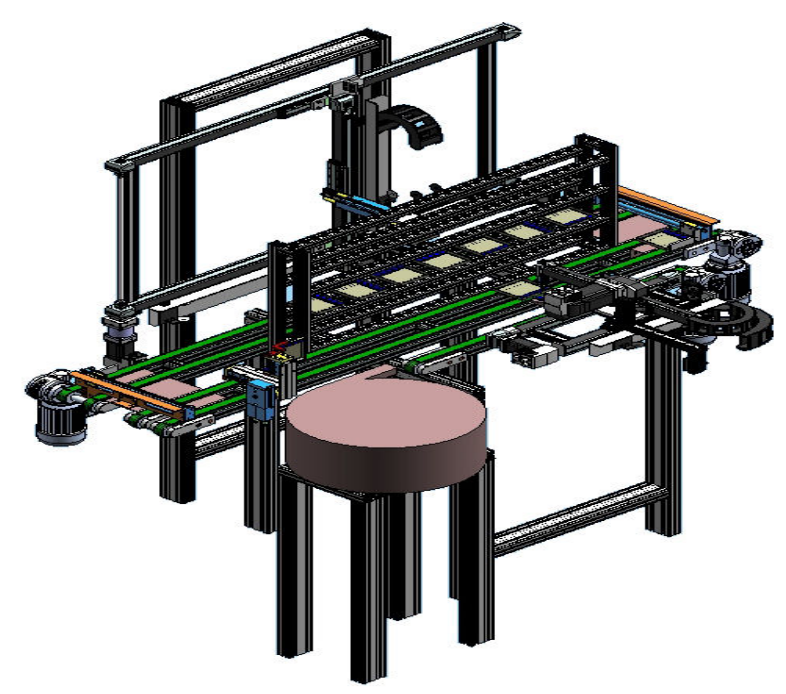

Fig. 4 Flexible manufacturing system model

Technological level of management in distributed system control method represents the lowest level in terms of hierarchy. Here, the control system directly affects the controlled system. Real-time control of FMS runs via the PLC controller (Programmable Logic Controller) that acquires data from sensors whereby data acquired from sensors represents inputs into control algorithms. Output values from the control algorithms are then via output modules of the PLC controller sent out to the actuators that represent drive unit of flexible manufacturing system. Actuators of this system are step motors, three phase motors and various kinds of pneumatic devices.

FMS is equipped with two three-axial manipulators from the company Schneider Electric, of which the first labelled as MAXP12R-H41BR-C41BR0300 serves for handling coloured cubes by means of vacuum chuck - to compile a colourful pattern onto a pallet; the second one labelled as MAXR12R-S41BR1000-P41BR0600 executes stacking finished pallets into the rack where it is possible to stack as many as 28 pallets. Both manipulators are driven in two axes by step motors and the third axis is 
driven by a pneumatic piston. A distinctive system is applied to both manipulators: the first one works horizontally and the second one works vertically. The manipulator for the shelf collator (vertical) unlike the first manipulator has one braked motor for security reasons. In case of power failure or if step motor power is cut off for any other reason, this motor is immediately braked and thus fall of the manipulator is avoided. Since more detailed description of FMS would not be appropriate for this article, more details regarding its description and the description of individual manipulators and their driving methods can be found in [1].

As can be seen in Fig. 5, all step motors used for driving the manipulators are connected to the PLC controller by means of the network DeviceNet. As a result, a DeviceNet communication card is connected to the PLC controller. These DeviceNet devices are connected to this card and thus they can communicate with the PLC controller in both directions.

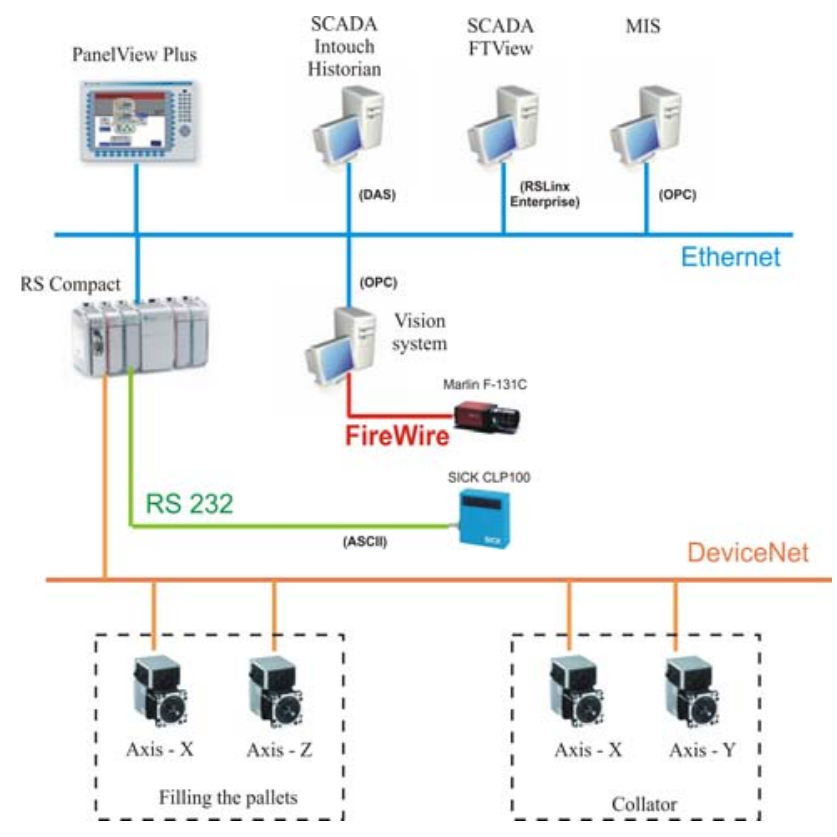

Fig. 5 FMS devices interconnection

Unlike the manipulators, three-phases motors are employed to set conveyors in motion. These motors are controlled by PLC digital outputs that are switched via three-phase relay.

We can also put a made-to-order shaking container manufactured by co. Vondra a Vondra (shown in Fig.1 at the bottom right) into the group of actuators. This container is used to supply the flexible manufacturing system with coloured plastic tubes. Using this device, cubes are gradually supplied from the container onto the conveyor belt in random colour order from where they are gradually taken away and sorted out by colour. Having said that, it is apparent that there are coloured cubes in the container, however, they are not sorted out in terms of colour.

Compiling pallets on the belt conveyor, moving from one conveyor to the other and tipping of pallets are realised by means of pneumatic elements, whereby pneumatics is also used as driving mechanism for fingers of the manipulator and motion of one of the axis of the manipulator. All pneumatic devices are controlled by digital electric signals from output cards of the PLC controller.

Most of the FMS sensors and actuators are connected directly to the input and the output cards of the PLC controller. For instance colour or presence monitoring sensors, end limit sensors of manipulators and other.

A number of sensors is employed in this system. The biggest part of them is made up of inductive and visual presence monitoring sensors and end position-monitoring sensors. They are distributed all over the FMS where they monitor motion of the pallets via individual posts on belt conveyors and they also check presence of objects in arms, end positions of the manipulators and their fingers and other.

A vision inspection system can also be regarded as a sensor or an individual post. The vision system is used to check a compiled pallet before it is placed in the collator. The heart of this post is the colour industrial vision camera Marlin F-131C whose light conditions are improved by two external light sources. As shown in Fig. 5, the camera is connected to the computer via FireWire interface that pocesses data from the camera and final values are provided to the control system and the information system (IS) by means of the Ethernet interface and the OPC protocol. This post is located at the end of one of the belt conveyors whereby a pallet gets out of the post by means of a pneumatic sliding device until it reaches the beginning of the second belt conveyor that can transport it as far as the rack collator. The pallet is transported into the rack only if its compiled pattern has been evaluated by the inspection system as correct. Otherwise, the pallet is emptied as soon as it has been evaluated by the camera and it must be compiled over again.

Sorting out the cubes by colours is allowed by a colour monitoring sensor Keyence labelled as CZ-H35S that is connected to the evaluation device Keyence labelled as CZ-V21AP whose output is connected to the PLC controller. This sensor recognizes 16 colours and it provides this 4-bit information further to the PLC controller. Based on data about the cube colour, the PLC controller can sort out cubes by colours by means of a special device shown in Fig. 6.

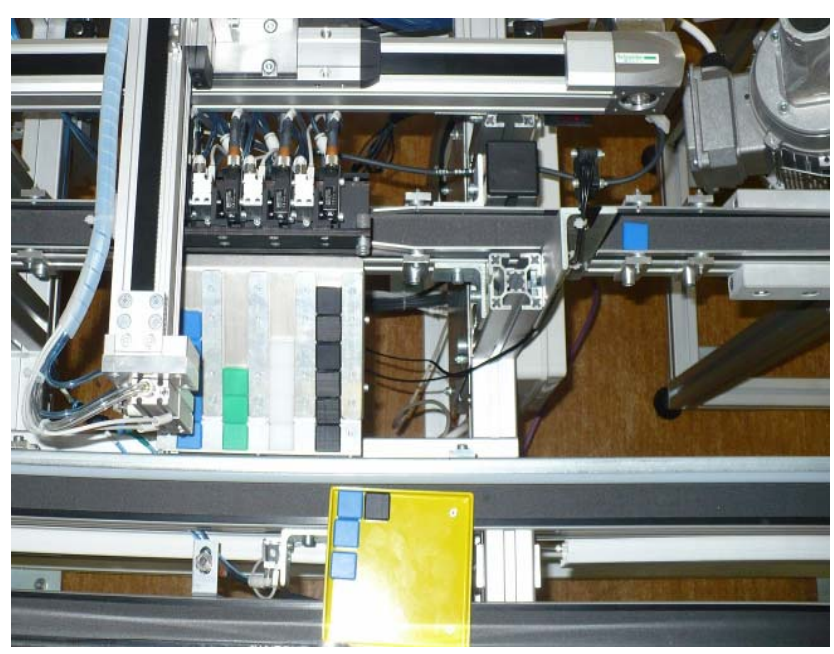

Fig. 6 Colour monitoring sensor with device to sort out cubes 
Colour monitoring sensor can be found at the top right in Fig. 6, located above the belt conveyor on which a blue cube is just coming and approaching the sensor. Left of the sensor, a pneumatic device that sorts the cubes out is located; a couple of sorted cubes in containers can be seen there. Over the container with blue cubes there is a manipulator arm with a vacuum chuck by which the manipulator puts cubes from the container onto the pallet (that yellow metal box).

A stationary CCD CLP 100 bar code scanner from Sick company also belongs to a group of devices labelled as sensors/scanners. This scanner reads the bar code of a pallet which is located on its bottom part. The scanner features high speed and can even read codes with High Density thanks to special lenses. In spite of small size, its performance is big enough. Extremely powerful decoder is integrated in this tiny bar code reader and transfers ASCII data via RS 232 interface into the PLC controller.

\subsection{Description of flexible manufacturing system activity}

Flexible manufacturing system (FMS) has been designed for production of colour patterns out of colour cubes that are placed into the pallets. There are cubes of four colours, namely blue, black, white and green. Individual patterns has the size of $5 \times 5$ cubes and their actual size is $2 \times 2 \mathrm{~cm}$. A request (it would be a purchase order in practice) comes from the information system (IS) in which individual incoming orders are processed. A production plan will be created in the IS; on production plan basis a request for production is then sent to the FMS control system.

Description:

1 - Post 1 - filling the pallets

2 - Post 2 - vision inspection, transfer between conveyors 1 and 2

3 - Post 3 - collating the pallets into the rack

4 - Post 4 - transfer between conveyors 2 and 1

5 - Post 5 -emptying the pallets

6 - Post 6 - sorting out cubes by colour

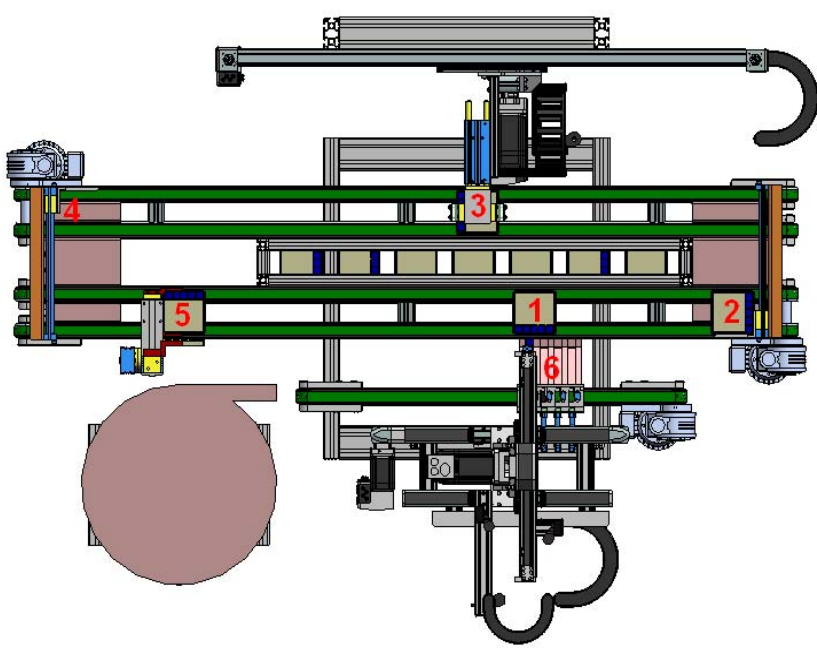

Fig. 7 Flexible manufacturing system posts

The first step in colour patterns production is sorting out the cubes. The cubes move from the shaking container along the belt conveyor to the cube sorting machine. There is a colour monitoring sensor on this conveyor. The sorting machine sorts the cubes out based on the information about the colour of the cube. It is possible to put 7 cubes of same colour in one container. When containers are full, the sorting process stops.

In the second step, a three-axial manipulator labelled as MAXP12R-H41BR-C41BR0300 compiles a desired pattern out of the cubes of corresponding colour onto the pallet. One pattern can contain maximum 25 colour cubes. Motion in $\mathrm{X}$ and $\mathrm{Z}$ axes of this manipulator is provided by step motors controlled by the PLC controller via the network DeviceNet. The third axis is controlled pneumatically. This motion is executed by a pneumatic piston that uplifts the cube. Holding the cube is provided by a vacuum chuck. A bar code scanned with bar code reader CLP 100 is assigned to the compiled pattern in the IS.

Having compiled the pattern, the pallet is transported by the conveyor to the next post where compiled pattern is inspected by the vision system. The vision system scans the compiled pattern and based on the recognition algorithm evaluates correctness of the compiled pattern according to a corresponding order. After vision inspection is completed, the pallet is moved to the second conveyor. When a compiled pattern does not match the order, the pallet is emptied in the following step, the content of the pallet is poured back to the shaking container and the IS evaluates this order as a failed order, and the request for creation of order will be made again.

In case the pattern has been compiled correctly, the pallet will be placed in the rack in the next step. Placement of the pallet in the rack is provided by a threeaxial manipulator labelled as MAXR12R-S41BR1000P41BR0600. This manipulator also takes pallets out of the rack. Step motors that control the motion of the manipulator in $\mathrm{X}$ and $\mathrm{Y}$ axes, are controlled via the network DeviceNet. The seating position of each compiled pallet with its bar code is also recorded in the IS.

If we want to empty the pallets placed in the rack, we use the manipulator again to take pallets out of the rack and put them on the belt conveyor. Selected pallets from the rack move to the end of the conveyor where they are transported onto the second conveyor by a pneumatic piston. There is a pneumatic dumping manipulator located at the post 5, which is at the beginning of the second conveyor. This manipulator pours the cubes from the pallet back to the shaking container.

\section{CONCLUSION}

Real models in the teaching process have a great motivational ability. It is a unique way how to gain practical experience with real control systems. The FMS model is of that kind and it is used in the teaching process where students can become acquainted with and get improved in various fields such as PLC controller programming, creation of SCADA/HMI applications, image recognition, database systems management, implementation of information systems and utilizing communication protocols. This system was constructed using standard industrial components and that means students can, as early as in the teaching process, get 
acquainted with systems that are actually implemented in industry. Thanks to this teaching method, graduates from our university will be able to prove themselves more competent at the labour market after finishing their studies.

\section{ACKNOWLEDGMENTS}

This contribution is the results of the Scientific Grant Agency of the Slovak Republic and of the project implementation: Centre of Information and Communication Technologies for Knowledge Systems (project number: 26220120020), supported by the Research and Development Operational Program funded by the ERDF.

\section{REFERENCES}

[1] JADLOVSKÝ, J. - LACIŇÁK, S. - CHOVAŇÁK, J. - ILKOVIČ, J.: Návrh distribuovaného systému riadenia pružnej výrobnej linky (Proposal of Distributed Control System of Flexible Production Line), Cybernetics and Informatics, Vyšná Boca, Slovak Republic, February 10-13, 2010, STU Bratislava.

[2] BALOGH, R. - BÉLAI, I. - DORNER, J. DRAHOŠ, P.: Priemyselné komunikácie (Industrial Communications), Slovak University of Technology in Bratislava, Slovak Republic, 2001, pp. 1-6, ISBN 80-227-1600-6.

[3] ČOPÍK, M.: Návrh a realizácia riadenia pružného výrobného systému (Proposal and Implementation of Flexible Production System Control), Diploma thesis, Technical University of Košice, Faculty of Electrical Engineering and Informatics, 2010, Slovak Republic.

Received November 8, 2010, accepted March 11, 2011

\section{BIOGRAPHIES}

Ján Jadlovský works at the Department of Cybernetics and Artificial intelligence of Technical University in
Košice as a pedagogue. He is a graduate of Technical University in Košice, Faculty of Electrical Engineering. In terms of pedagogy he focuses on the issues of proposal and implementation of distributed systems that control production processes. In his science-research based activities he is oriented towards distributed control systems, image recognition, complex functional diagnostics of single purpose regulators, diagnostics of production control systems, creation of information and control systems with application of the latest information technology. He is a chief executive of company KYBERNETIKA, s.r.o., Košice that is oriented towards design engineering, implementation and operation of production and diagnostic systems in the electrical engineering, mechanical and metallurgical production.

Stanislav Laciňák was born on 19.12.1978. He graduated with distinction (MSc) from Technical University of Košice, the Department of Cybernetics and Artificial Intelligence of the Faculty of Electrical Engineering and Informatics in 2002. He completed a dissertation Analysis and proposal of complex network control systems in 2006. $\mathrm{He}$ is currently a lecturer at the Technical University. In his science-research based activities he is oriented towards distributed control systems using industrial networks and diagnostics of control systems by means of Petri Nets.

Matej Čopík was born on 2.3.1986. In 2010 he graduated (MSc) with distinction of Department of Cybernetics and Artificial Intelligence of the Faculty of Electrical Engineering and Informatics at Technical University in Košice. Since 2010 he is working as a $\mathrm{PhD}$ student at the Department of Cybernetics and Artificial Intelligence at Technical University in Košice. His scientific research is focusing on methodology for the design and implementation of production robototechnologys lines.

Ján Ilkovič was born on 17.9.1984. In 2009 he graduated MSc degree at department of Cybernetics and Artificial Intelligence of the Faculty of Electrical Engineering and Informatics at Technical University in Košice. Since 2009 he is a PhD. student at the Department of Cybernetics and Artificial Intelligence. His scientific is focusing on modelling and diagnosis of handling and robotic systems. 